\title{
Studying Kidney Diseases at the Single-Cell Level
}

\author{
Mengmeng Jiang ${ }^{a, b}$ Haide Chen ${ }^{b, c}$ Guoji Guo ${ }^{a, b, c, d, e}$ \\ aLiangzhu Laboratory, Zhejiang University Medical Center, Hangzhou, China; ${ }^{b}$ Center for Stem Cell and \\ Regenerative Medicine, Zhejiang University School of Medicine, Hangzhou, China; 'Zhejiang Provincial Key \\ Lab for Tissue Engineering and Regenerative Medicine, Dr. Li Dak Sum \& Yip Yio Chin, Center for Stem Cell and \\ Regenerative Medicine, Hangzhou, China; 'Bone Marrow Transplantation Center, The First Affiliated Hospital,

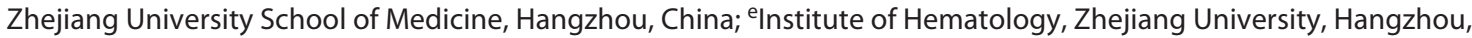 \\ China
}

\section{Keywords}

Single-cell technology · Kidney disease - Immune cell ·

Kidney organoid · Allograft

\section{Abstract}

Background: The kidney is a highly complex organ that performs diverse functions that are essential for health. Kidney disease occurs when the kidneys are damaged and fail to function properly. Single-cell analysis is a powerful technology that provides unprecedented insights into normal and abnormal kidney cell types and will transform our understanding of the mechanism underlying common kidney diseases. Summary: Our understanding of kidney disease pathogenesis is limited by the incomplete molecular characterization of cell types responsible for kidney functions. Application of single-cell technologies for the study of the kidney has revealed cellular heterogeneity, gene expression signatures, and molecular dynamics during the onset and development of kidney diseases. Single-cell analyses of kidney organoids and allograft tissues offer new insights into kidney organogenesis, disease mechanisms, and therapeutic outcomes. Collectively, a better understanding of kidney cell heterogeneity and the molecular dynamics of kidney diseases will improve diagnostic accuracy and facilitate the identification of novel treatment strategies in nephrology. Key Message: In this review article, we summarize recent single-cell studies on kidney diseases and discuss the impact of single-cell technology on both basic and clinical nephrology research.

(C) 2021 The Author(s)

Published by S. Karger AG, Basel

\section{Introduction}

The kidneys are 2 bean-shaped organs that are responsible for filtering waste products, excess water, and other impurities from the blood and producing urine. The kidneys also regulate $\mathrm{pH}$, salt, potassium levels, and blood pressure; control the production of red blood cells; and activate a form of vitamin $\mathrm{D}$ that helps the body absorb calcium $[1,2]$. To date, an estimated 850 million people worldwide have kidney diseases, including chronic kidney diseases (CKD), acute kidney injury, kidney failure, and many other diseases [3]. Kidney disease occurs when the kidneys are damaged and cannot perform their function. Damage may be caused by diabetes, high blood pres-

Correspondence to:

karger@karger.com www.karger.com/kdd

\section{Published by S. Karger AG, Basel}

This is an Open Access article licensed under the Creative Commons Attribution-NonCommercial-4.0 International License (CC BY-NC) (http://www.karger.com/Services/OpenAccessLicense), applicable to the online version of the article only. Usage and distribution for commercial purposes requires written permission. 


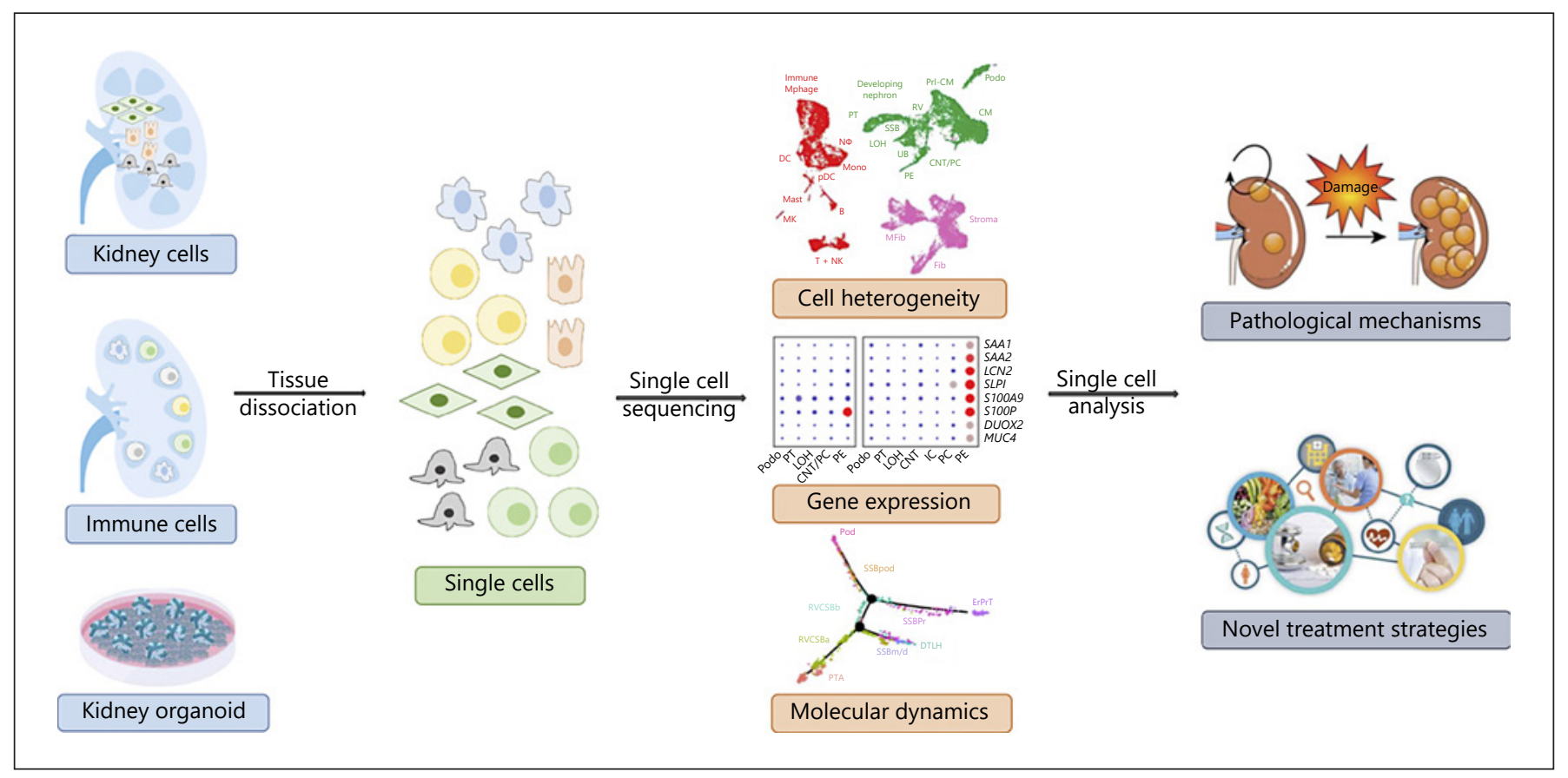

Fig. 1. Studying kidney diseases at the single-cell level. The application of single-cell technologies to the study of diseased kidney cells, as well as immune cells in the tissues and kidney organoids, reveals cellular heterogeneity, gene expression signatures, and molecular dynamics during the onset and development of kidney diseases, facilitating the identification of pathological mechanisms and novel treatment strategies in nephrology.

sure, and various other chronic (long-term) conditions. Kidney diseases can lead to other health problems, including weak bones, nerve damage, malnutrition, and heart disease. The current therapeutic strategies for patients still involve kidney transplantation or dialysis, which are costly $[2,3]$.

A variety of cells in the kidney, including epithelial, mesangial, endothelial, and neuronal cells, as well as a network of immune cells, interact to maintain normal kidney function. Insights into the heterogeneity of healthy kidneys and the process underlying kidney diseases will refine kidney molecular and histopathological phenotype definitions and support the development of new disease classifications. Single-cell technology has potential advantages for the determination of cell subtypes, states, and frequency changes during kidney disease onset and progression [4]. With the rapid development of highthroughput single-cell RNA sequencing (scRNA-seq), comprehensive cellular landscapes of normal kidneys have recently been constructed for precision medicine in nephrology [5-9]. The Kidney Precision Medicine Project (KPMP) was developed worldwide, aiming to obtain human kidney biopsies, create a kidney tissue atlas, define disease subgroups, and eventually identify critical cells, pathways, and targets for novel therapies [10]. On the basis of the relevant single-cell transcription datasets for the kidney, researchers have also analyzed the gene expression signatures of ACE2, TMPRSS2, and SLC6A19 in kidney cell subtypes, which is critical for understanding the pathogenesis of severe acute respiratory syndrome coronavirus 2 [11]. In this review, we will focus on (1) the development and application of single-cell technologies, (2) studying the occurrence and development of kidney diseases using scRNA-seq, (3) a molecular atlas of immune cells in kidney diseases, (4) the application of scRNA-seq on kidney organoids, and (5) insight into kidney allografts by scRNA-seq (Fig. 1).

\section{Development and Application of Single-Cell Transcriptomic Technology}

Individual cells are fundamental units of life. Singlecell profiling technologies revolutionize our ability to identify cellular compositions, track molecular dynamics, and reveal pathological mechanisms. In the early 
stages, microarray and qPCR technologies were employed for global single-cell gene expression analysis. Tietjen et al. [12] monitored the molecular expression profiles of individual neurons and progenitor cells and defined signaling pathways at a distinct developmental stage using a single-cell microarray. In addition, singlecell expression analysis identified several subtypes of developing cells with novel pancreatic genes, providing new insights into pancreas development [13]. Our group developed single-cell qPCR technology and applied it to investigate key regulatory genes involved in mouse blastocyst development and hematopoietic lineage differentiation $[14,15]$.

With the advent of next-generation sequencing technology, scRNA-seq exhibited obvious advantages over microarrays in distinguishing different isoforms, allelic expression, and new transcripts in single cells at a low cost [16]. In 2009, Tang et al. [17] first reported mRNA wholetranscriptome sequencing of a single cell, demonstrating the complexity of transcript variants in individual mouse blastomeres or oocytes. Smart-seq, which detects fulllength transcripts in single cells, was developed in 2012, and by applying it to rare cells, researchers identified candidate biomarkers for melanoma circulating tumor cells. One year later, Smart-seq2 with improved reverse transcription, read coverage, bias, and accuracy was introduced. The latest development of Smart-seq 3 greatly increased its sensitivity in detecting thousands of single-cell transcripts at allele and isoform resolution [18-20]. Unlike PCR-based amplification methods, CEL-seq captures efficient single-cell transcriptomics via multiplexed linear amplification. A study of early C. elegans embryonic development by CEL-seq revealed its reproducible and sensitive results at single-cell resolution $[21,22]$. The first automatic platform for scRNA-seq is Fluidigm $\mathrm{C}$, which traps single cells in 96 or 384 chambers using a microfluidic system, followed by cell lysis, reverse transcription, and PCR amplification [23].

Since 2015, with the continuous advent of Drop-seq, inDrop, 10× Genomics, Seq-well, Microwell-seq, SPLiTseq, and other technologies, single-cell research has completely entered the era of high-throughput, low cost, and automation. Drop-seq and inDrop separate individual cells into nanoliter-sized aqueous droplets and mix them with a unique barcode to label each cell $[24,25]$. On the other hand, Cyto-seq, Seq-well, and Microwell-seq achieve high-throughput single-cell mRNA sequencing by capturing one cell and one barcoded bead in microwells $[26,27]$. Our group constructed the first mouse cell atlas and human cell landscape at the single-cell level us- ing Microwell-seq [5, 8]. Most recently, more highthroughput and simpler methods, called SPLiT-seq and sci-RNA-seq for scRNA-seq, have been created $[28,29]$. These methods use cells or nuclei themselves as the reaction chamber through several rounds of split-and-pool for barcoding to label cells. All cells are then pooled for cDNA PCR amplification and sequencing. Cao et al. [30, 31] profiled the transcriptomes of approximately 2 million mouse organogenesis cells and 4 million human fetal cells using sci-RNA-seq3, providing a global view of mammalian developmental processes. Other single cell technologies covering genome, proteome and epigenome analyses have also flourished; but they are beyond the scope of this review.

\section{Occurrence and Progression of Kidney Disease}

The kidney can be affected by a number of prevalent and serious conditions, including acute kidney injury, glomerulonephritis, ascending infection (pyelonephritis), and cancer. In general, our understanding of kidney disease pathogenesis is limited by an incomplete molecular characterization of the cell types responsible for the organ's specific functions. To narrow this knowledge gap, our group and Park et al. [6] constructed transcriptional maps of healthy mouse kidneys using scRNA-seq. Major subtypes of nephron epithelial cells, including podocytes, proximal tubule epithelial cells, the loop of Henle, distal tubule, and collecting duct cells, were identified $[5,6]$. Research characterized a novel transitional cell type in collecting duct cell populations, confirming the findings in a previous scRNA-seq study of interconversion between intercalated and principal cells [32]. Moreover, Ransick et al. [33] profiled male and female adult kidneys and generated an anatomical atlas of mouse nephrons at the single-cell level. Our group has also performed Microwell-seq analyses of human fetal and adult kidney tissues. In addition to epithelial, endothelial, stromal, and tissue-resident immune cells, we found previously undescribed types of S-shaped body cells in the fetal kidney and a new transitional cell type in the adult kidney [8]. A single-cell molecular landscape of the kidney vasculature has revealed specialized expression signatures for building nephrons and has uncovered the pathogenesis of kidney disorders [34]. Furthermore, all cell types of kidney glomeruli from healthy mice and different disease models were identified using single-cell transcriptomic cluster analysis, and novel disease-related genes and regulatory pathways in disease models, such as the activated Hippo 
pathway in podocytes after nephrotoxic immune injury, have been detected [35].

Kidney fibrosis is the hallmark of CKD, which affects more than $10 \%$ of the world population. In parabiosis models of kidney fibrosis, Kramann et al. [36] confirmed that monocytes contributed a small population of myofibroblasts using scRNA-seq, but that most myofibroblasts were derived from mesenchymal cells. Subsequently, Kuppe et al. [37] profiled approximately 135,000 transcriptomes of cells from the proximal and non-proximal tubules and further verified distinct subtypes of mesenchymal cells as the main contributors to fibrosis in the human kidney. Moreover, in a comparative analysis of healthy and fibrotic kidney single cells, a myofibroblastspecific gene, naked cuticle homolog 2 (NKD2), was identified as a potential therapeutic target in human kidney fibrosis [37]. On the basis of 402 kidney biopsies, urinary fibrinogen has been predicted to be a noninvasive biomarker in patients with CKD [38].

Diabetic nephropathy (DN) is characterized by damage to both the glomerulus and tubulointerstitium. However, relatively little is known about how cell states and frequencies change with disease onset and progression with regard to gene expression. To elucidate glomerular cell gene expression changes in mouse DN, Fu et al. [39] performed scRNA-seq analysis and identified several new potential markers of glomerular cells, including Magi2, Robo2, Ramp3, and Fabp4. Regulation of angiogenesis and migration pathways were altered in endothelial cells, while pathways of regulation of translation and protein stabilization were highly enriched in mesangial cells in diabetic kidney diseases (DKDs). Overall, molecular dynamic changes in endothelial and mesangial cells will help in identifying important pathophysiologic factors contributing to the progression of DN. Chung et al. [35] profiled the single-cell transcriptome of the glomerulus in a mouse diabetes model. Gene expression changes of mesangial cells and podocytes in $o b / o b$ mice compared to control mice were revealed. Proliferation pathways were induced in mesangial cells and cell death-associated pathways were induced in podocytes, coinciding with the cell number proportional changes [35]. Integrated analysis of published DKD scRNA-seq datasets identified 17 hub genes, enriching our understanding of the molecular mechanisms underlying the pathogenesis of DKDs [40]. Moreover, unbiased single-nucleus RNA sequencing (snRNA-seq) has been performed on cryopreserved human diabetic kidney samples, generating 23,980 transcriptomes from 3 control and 3 early DN samples [41]. The results demonstrated cell type-specific changes in gene expression and suggested increased potassium secretion in human DN. A previous study comparing scRNA-seq and snRNA-seq of adult mouse kidneys showed a more effective capture capability for the latter. For instance, glomerular podocytes, mesangial cells, and endothelial cells were captured by snRNA-seq but not by scRNA-seq [42]. The utility of snRNA-seq minimizes enzymatic dissociation artifacts and can be performed on frozen samples, which is expected to lead to broader applications to accelerate studies of pathological mechanisms in diverse kidney diseases.

Precise cellular transcriptomes may reveal a kidney tumor cell of origin as well as the transcriptional trajectories underpinning malignant transformation. Young et al. [43] defined normal and cancerous human kidney cell types from a catalog of 72,501 single-cell transcriptomes. By identifying specific normal cell correlates of renal cancer cells (RCCs), a study provided evidence for the hypothesis that Wilms' tumor cells were aberrant fetal cells and RCCs might be derived from a little-known subtype of proximal convoluted tubule cells. Hence, scRNA-seq provides a scalable experimental strategy for determining the characteristics of human kidney cancer cells at a precise cellular, quantitative molecular resolution.

\section{Molecular Atlas of Immune Cells in Kidney Disease}

Immune cells are a foundational component of human tissues, playing a vital role in physiological and pathological metabolism. Indeed, the ability of the immune system to recognize and respond to pathogenic or danger signals, or to malignant cells, is critical. The application of single-cell technologies to the study of immune cells in the kidney has the potential to facilitate a better understanding of the role of the immune system in healthy kidneys and disease pathogenesis, as well as the identification of novel treatment strategies. These efforts are beginning to map the complex immune landscape within the kidney and reveal the relationship between tissue-resident immune cells and their inter-activated neighboring cells.

In 2018, the application of scRNA-seq to mouse kidney tissues provided a comprehensive immune cell landscape, including resident macrophages, neutrophils, B and T lymphocytes, and NK cells [6]. One year later, unprecedented single-cell insights into the spatiotemporal organization of human kidney immune cells were established. A network of tissue-resident myeloid and lymphoid immune cells in fetal and adult kidneys was re- 
vealed, and research identified postnatal acquisition of transcriptional programs that promoted infection-defense capabilities. In addition, cross talk between epithelial and immune cells in the mature kidney was predicted to recruit antibacterial macrophages and neutrophils to regions of the kidney that were most susceptible to infection. Overall, the comprehensive immune landscape of the kidney facilitates the study of pathogenic mechanisms and the identification of therapeutic targets in immune and infectious kidney diseases [44].

Lupus nephritis (LN) is a potentially fatal autoimmune disease for which the current treatment is ineffective and often toxic. The molecular and cellular processes that lead to renal damage and the heterogeneity of LN are still ambiguous. Arazi et al. [45] identified 21 disease-specific subsets of myeloid cells, T cells, natural killer cells, and B cells in patients with LN using scRNA-seq. Similarly, Der et al. $[46,47]$ applied scRNA-seq to renal and skin biopsy tissues from patients with LN. It has been reported that type I interferon response scores were higher in tubular epithelial cells from LN patients than in those from healthy controls. Furthermore, an inflammation and fibrotic signature of tubular cells was associated with failure to respond to treatment $[46,47]$. In DKD, scRNA-seq analysis revealed a significantly higher number of immune cells in diabetic glomeruli than in the control [39]. These studies show that the gene expression profiles of immune cells in urine, skin, and kidney are highly relevant, suggesting that urine and skin biopsies might serve as potential sources of diagnostic and prognostic markers for renal diseases.

Recent studies have shown the role of immune cells in kidney disease models at single-cell resolution. The unilateral ureteral obstruction model is widely used in renal interstitial fibrosis, resulting in marked renal hemodynamic and metabolic changes with infiltration of macrophages and inflammatory cells into the renal interstitium. scRNA-seq has been employed in a reversible unilateral ureteral obstruction model to dissect the myeloid cell landscape during the progression and regression of fibrosis. Conway et al. [48] revealed myeloid cell heterogeneity, and the relative proportions of the subsets changing dynamically, with monocytes being recruited in early injury, $\mathrm{Cr} 2^{+}$macrophages accumulating in late injury, and a novel $M m p 12^{+}$macrophage cluster acting during repair. Ischemia-reperfusion injury (IRI) is linked to inflammation and leukocyte recruitment in kidney diseases and transplantation. An experimental model of IRI was used to assess the functional role of group 2 innate lymphoid cells in the kidney, and the data suggested a redundant function for these cells in renal injury [49]. By applying scRNA-seq to a kidney transplantation IRI model, Kreimann et al. [50] found increased levels of systemic CXCL13 with subsequent infiltration of CXCR5 ${ }^{+}$ leukocytes. Kirita et al. [51] also applied snRNA-seq to mouse models of IRI to describe detailed cellular responses after injury and identified a distinct pro-inflammatory and pro-fibrotic proximal tubule cell state. Uric acid is the final oxidation product of purine metabolism, with a strong association with renal inflammation across several disease models. For instance, NLRP3 (NOD-, LRR-, and pyrin domain-containing 3 ) senses the signal of excessive uric acid, and the inflammasome is activated in hyperuricemic nephropathy [52]. However, the specific immune mechanisms of hyperuricemia-induced renal injury remain unclear. Construction of the inflammasome landscape at the single-cell level in hyperuricemia-induced renal injury models is expected in the near future.

Immune infiltrates are present within kidney tumors and are altered by the tumor microenvironment. A previous study revealed a population of tumor-infiltrating macrophages expressing vascular endothelial growth factor A and participating in a complex VEGF signaling circuit in RCC tissues [43]. This study illustrated the ability of scRNA-seq to address questions of tumor ontogeny and to identify putative pathophysiological mechanisms and cell signaling networks that may serve as targets for pharmacological treatment. Collectively, research has highlighted the advancement of scRNAseq to provide insights into the immune system and the cellular networks that operate in healthy and diseased kidneys.

\section{Application of scRNA-seq to Kidney Organoids}

In addition to studies of diseased kidney tissues, kidney organoids have emerged as critical tools to investigate organogenesis and disease mechanisms, and potentially as a replacement tissue source to expedite therapeutic development. In parallel, advancesin scRNA-seq have led to more detailed analyses of a variety of cell subgroups and gene expression changes in kidney organoids [53].

In general, a better understanding of kidney embryonic development at the single-cell level is necessary to guide the maturation of kidney organoids. A single-cell transcriptomics study of the human fetal kidney identified 22 cell types and corresponding marker genes [7]. 
Comparison of different developmental stages revealed continuous molecular dynamics in podocytes. Another single-cell analysis was performed on human fetal kidneys and embryonic stem cell-derived kidney organoids [54]. Comparative analysis of kidney single-cell developmental trajectories showed similar gene expression signatures in vivo and in vitro, except for late mature podocytes, suggesting an incomplete maturation process of podocyte organoids. Transplantation experiments have further indicated that optimization of the mesangial and vascular compartments can be performed using organoid models. A recent study used metanephric mesenchyme and ureteric bud-like cells to generate kidney organoids, revealing improved proximal tubule maturation and reduced off-target cell populations by scRNA-seq [55]. Consistently, Subramanian et al. [56] and $\mathrm{Wu}$ et al. [57] profiled human pluripotent stem cell (PSC)-derived kidney organoids by scRNA-seq. Compared to human fetal and adult kidney single-cell datasets, different kidney organoids displayed largely reproducible cell types but variable cell proportions because of off-target cells. Moreover, transcription factor network analysis uncovered the kidney organoid differentiation pathway, highlighting the power of single-cell technologies in characterizing and directing organoid differentiation.

Together, human kidney organoids are useful resources for mining disease models, underlying regulatory mechanisms, high-throughput drug screening, and ultimately regenerative therapy $[58,59]$. These studies emphasize the potential utility of scRNA-seq in kidney model systems to elucidate in vivo physiological and pathological processes and provide guidance for future studies on kidney disease diagnosis and treatment.

\section{Insight into Kidney Allografts by scRNA-seq}

Allografts are one of the most effective methods in the clinical therapy of end-stage kidney diseases. Single-cell technologies offer opportunities to describe kidney cell types and states with accuracy and precision using a human biopsy specimen after allograft transplantation. The first published report of kidney allograft biopsy specimens profiled 8,746 single-cell transcriptomes and defined a diverse inflammatory response. Monocytes formed a nonclassical CD $16^{+}$group and a classic CD16 ${ }^{-}$ group; endothelial cells presented a resting state and 2 antibody-mediated rejection response states. These findings help us better understand immune rejection in kidney transplantation [60]. In a comparative study of re- cipient and donor-origin kidney single cells, distinct transcriptional gene expression of kidney biopsy specimens was profiled. Inflammatory activated macrophages and cytotoxic-expressed $\mathrm{T}$ cells in recipients were observed [61]. Similarly, Liu et al. [62] analyzed cells from chronic kidney transplant rejection and matched healthy adult kidneys at the single-cell level. Unsupervised clustering analysis revealed that increased numbers of immune cells and myofibroblasts may lead to renal rejection and fibrosis in the chronic kidney transplant rejection group [62]. Remarkably, a recent study profiled the first single-cell atlas of adult human urine and identified a $\mathrm{SOX}^{+}$kidney stem/progenitor cell population in the urine. Progenitor cells successfully proliferated and differentiated in vivo and gained some properties of tubular cells, providing a potentially useful resource for future kidney transplantation therapy [63]. Collectively, scRNA-seq technologies provide novel and deep insights into human renal transplantation rejection, ultimately improving diagnostic accuracy and accelerating the adoption of molecular biopsy interpretation.

\section{Conclusion}

Over the past decade, scRNA-seq has become an indispensable tool for transcriptome-wide analysis of differential gene expression to investigate physiological biology and determine the molecular dysregulation in diseases [64]. Unlike traditional discrete phenotypes, molecular features at the single-cell level may offer a systematic standard for describing kidney disease phenotypes. The application of single-cell technologies to healthy kidney tissues or clinical renal biopsy samples has provided comprehensive kidney molecular atlases and broadens our understanding in the nephrology. Single-cell atlases of the kidney will be an integral part of the international effort on the Human Cell Atlas, which aims at producing a comprehensive and systematic reference map of the human body [65]. In future studies, single-cell ultrahighthroughput and spatial transcriptomic analyses are expected to expand our understanding of kidney. The integration of multi-omics data will further improve personalized diagnoses and treatments for kidney diseases.

\section{Conflict of Interest Statement}

The authors declare that they have no competing interests. 


\section{Funding Sources}

This work was supported by the National Natural Science Foundation of China (31930028, 91842301, 81770188, and 82090012 ) and the National Key Research and Development Program of China (2018YFA0107804, 2018YFA0107801, and 2018YFA0800503).

\section{Author Contributions}

Mengmeng Jiang drafted the literature. Haide Chen and Guoji Guo provided valuable input into the finalization of the article. All authors read and approved the final manuscript.

\section{References}

1 Kriz W, Bankir L. A standard nomenclature for structures of the kidney. The renal commission of the international union of physiological sciences (IUPS). Kidney Int. 1988 Jan; 33(1):1-7.

2 Kurts C, Panzer U, Anders HJ, Rees AJ. The immune system and kidney disease: basic concepts and clinical implications. Nat Rev Immunol. 2013 Oct;13(10):738-53.

3 Luyckx VA, Al-Aly Z, Bello AK, BellorinFont E, Carlini RG, Fabian J, et al. Sustainable development goals relevant to kidney health: an update on progress. Nat Rev Nephrol. 2021 Jan;17(1):15-32.

4 Clark AR, Greka A. The power of one: advances in single-cell genomics in the kidney. Nat Rev Nephrol. 2020 Feb;16(2):73-4.

5 Han X, Wang R, Zhou Y, Fei L, Sun H, Lai S, et al. Mapping the mouse cell atlas by microwell-seq. Cell. 2018 Feb;172(5):1091-107.e17.

6 Park J, Shrestha R, Qiu C, Kondo A, Huang S, Werth M, et al. Single-cell transcriptomics of the mouse kidney reveals potential cellular targets of kidney disease. Science. 2018 May; 360(6390):758-63.

7 Hochane $M$, van den Berg PR, Fan X, Bérenger-Currias N, Adegeest E, Bialecka M, et al. Single-cell transcriptomics reveals gene expression dynamics of human fetal kidney development. PLoS Biol. 2019 Feb; 17(2): e3000152.

8 Han X, Zhou Z, Fei L, Sun H, Wang R, Chen $\mathrm{Y}$, et al. Construction of a human cell landscape at single-cell level. Nature. 2020 May; 581(7808):303-9.

9 Liao J, Yu Z, Chen Y, Bao M, Zou C, Zhang H, et al. Single-cell RNA sequencing of human kidney. Sci Data. 2020 Jan;7(1):4.

10 Precision medicine in nephrology. Nat Rev Nephrol. 2020 Nov;16(11):615.

11 Chen QL, Li JQ, Xiang ZD, Lang Y, Guo GJ, Liu ZH. Localization of cell receptor-related genes of SARS-CoV-2 in the kidney through single-cell transcriptome analysis. Kidney Dis. 2020 Jul;6(4):258-70.

12 Tietjen I, Rihel JM, Cao Y, Koentges G, Zakhary L, Dulac C. Single-cell transcriptional analysis of neuronal progenitors. Neuron. 2003 Apr;38(2):161-75.

13 Chiang MK, Melton DA. Single-cell transcript analysis of pancreas development. Dev Cell. 2003 Mar;4(3):383-93.
14 Guo G, Huss M, Tong GQ, Wang C, Li Sun L, Clarke ND, et al. Resolution of cell fate decisions revealed by single-cell gene expression analysis from zygote to blastocyst. Dev Cell. 2010 Apr;18(4):675-85.

15 Guo G, Luc S, Marco E, Lin TW, Peng C, Kerenyi MA, et al. Mapping cellular hierarchy by single-cell analysis of the cell surface repertoire. Cell Stem Cell. 2013 Oct;13(4):492505.

16 Wang Z, Gerstein M, Snyder M. RNA-Seq: a revolutionary tool for transcriptomics. Nat Rev Genet. 2009 Jan;10(1):57-63.

17 Tang F, Barbacioru C, Wang Y, Nordman E, Lee $\mathrm{C}, \mathrm{Xu} \mathrm{N}$, et al. mRNA-Seq whole-transcriptome analysis of a single cell. Nat Methods. 2009 May;6(5):377-82.

18 Ramsköld D, Luo S, Wang YC, Li R, Deng Q, Faridani OR, et al. Full-length mRNA-seq from single-cell levels of RNA and individual circulating tumor cells. Nat Biotechnol. 2012 Aug;30(8):777-82.

19 Picelli S, Björklund ÅK, Faridani OR, Sagasser S, Winberg G, Sandberg R. Smart-seq2 for sensitive full-length transcriptome profiling in single cells. Nat Methods. 2013 Nov; 10(11): 1096-8.

20 Hagemann-Jensen M, Ziegenhain C, Chen P, Ramsköld D, Hendriks GJ, Larsson AJM, et al. Single-cell RNA counting at allele and isoform resolution using Smart-seq3. Nat Biotechnol. 2020 Jun;38(6):708-14.

21 Hashimshony T, Wagner F, Sher N, Yanai I. CEL-seq: single-cell RNA-seq by multiplexed linear amplification. Cell Rep. 2012 Sep;2(3): 666-73.

22 Yanai I, Hashimshony T. CEL-seq2-singlecell RNA sequencing by multiplexed linear amplification. Methods Mol Biol. 2019;1979: 45-56.

23 Streets AM, Zhang X, Cao C, Pang Y, Wu X, Xiong L, et al. Microfluidic single-cell wholetranscriptome sequencing. Proc Natl Acad Sci U S A. 2014 May;111(19):7048-53.

24 Klein AM, Mazutis L, Akartuna I, Tallapragada N, Veres A, Li V, et al. Droplet barcoding for single-cell transcriptomics applied to embryonic stem cells. Cell. 2015 May;161(5): 1187-201.

25 Macosko EZ, Basu A, Satija R, Nemesh J, Shekhar K, Goldman M, et al. Highly parallel genome-wide expression profiling of individual cells using nanoliter droplets. Cell. 2015 May;161(5):1202-14.
26 Fan HC, Fu GK, Fodor SP. Expression profiling. Combinatorial labeling of single cells for gene expression cytometry. Science. 2015 Feb; 347(6222): 1258367.

27 Gierahn TM, Wadsworth MH, Hughes TK, Bryson BD, Butler A, Satija R, et al. Seq-well: portable, low-cost RNA sequencing of single cells at high throughput. Nat Methods. 2017 Apr;14(4):395-8.

28 Cao J, Packer JS, Ramani V, Cusanovich DA, Huynh C, Daza R, et al. Comprehensive single-cell transcriptional profiling of a multicellular organism. Science. 2017 Aug;357(6352): 661-7.

29 Rosenberg AB, Roco CM, Muscat RA, Kuchina A, Sample P, Yao Z, et al. Single-cell profiling of the developing mouse brain and spinal cord with split-pool barcoding. Science. 2018 Apr;360(6385):176-82.

30 Cao J, Spielmann M, Qiu X, Huang X, Ibrahim $\mathrm{DM}$, Hill AJ, et al. The single-cell transcriptional landscape of mammalian organogenesis. Nature. 2019 Feb;566(7745):496-502.

31 Cao J, O’Day DR, Pliner HA, Kingsley PD, Deng M, Daza RM, et al. A human cell atlas of fetal gene expression. Science. 2020; 370(6518):370.

32 Chen L, Lee JW, Chou CL, Nair AV, Battistone MA, Păunescu TG, et al. Transcriptomes of major renal collecting duct cell types in mouse identified by single-cell RNA-seq. Proc Natl Acad Sci U S A. 2017 Nov;114(46): E9989-98.

33 Ransick A, Lindström NO, Liu J, Zhu Q, Guo JJ, Alvarado GF, et al. Single-cell profiling reveals sex, lineage, and regional diversity in the mouse kidney. Dev Cell. 2019 Nov;51(3):399_ 413.e7.

34 Barry DM, McMillan EA, Kunar B, Lis R, Zhang T, Lu T, et al. Molecular determinants of nephron vascular specialization in the kidney. Nat Commun. 2019 Dec;10(1):5705.

35 Chung JJ, Goldstein L, Chen YJ, Lee J, Webster JD, Roose-Girma M, et al. Single-cell transcriptome profiling of the kidney glomerulus identifies key cell types and reactions to injury. J Am Soc Nephrol. 2020 Oct;31(10): 2341-54.

36 Kramann R, Machado F, Wu H, Kusaba T, Hoeft K, Schneider RK, et al. Parabiosis and single-cell RNA sequencing reveal a limited contribution of monocytes to myofibroblasts in kidney fibrosis. JCI Insight. 2018 May;3(9): e99561. 
37 Kuppe C, Ibrahim MM, Kranz J, Zhang X, Ziegler S, Perales-Patón J, et al. Decoding myofibroblast origins in human kidney fibrosis. Nature. 2021 Jan;589(7841):281-6.

38 Wang $\mathrm{H}$, Zheng C, Lu Y, Jiang Q, Yin R, Zhu $\mathrm{P}$, et al. Urinary fibrinogen as a predictor of progression of CKD. Clin J Am Soc Nephrol. 2017 Dec;12(12):1922-9.

$39 \mathrm{Fu}$ J, Akat KM, Sun Z, Zhang W, Schlondorff D, Liu Z, et al. Single-cell RNA profiling of glomerular cells shows dynamic changes in experimental diabetic kidney disease. J Am Soc Nephrol. 2019 Apr;30(4):533-45.

40 Zhang Y, Li W, Zhou Y. Identification of hub genes in diabetic kidney disease via multiplemicroarray analysis. Ann Transl Med. 2020 Aug;8(16):997.

41 Wilson PC, Wu H, Kirita Y, Uchimura K, Ledru N, Rennke HG, et al. The single-cell transcriptomic landscape of early human diabetic nephropathy. Proc Natl Acad Sci U S A. 2019 Sep;116(39):19619-25.

$42 \mathrm{Wu} \mathrm{H}$, Kirita Y, Donnelly EL, Humphreys $\mathrm{BD}$. Advantages of single-nucleus over singlecell RNA sequencing of adult kidney: rare cell types and novel cell states revealed in fibrosis. J Am Soc Nephrol. 2019 Jan;30(1):23-32.

43 Young MD, Mitchell TJ, Vieira Braga FA, Tran MGB, Stewart BJ, Ferdinand JR, et al. Single-cell transcriptomes from human kidneys reveal the cellular identity of renal tumors. Science. 2018 Aug;361(6402):594-9.

44 Stewart BJ, Ferdinand JR, Young MD, Mitchell TJ, Loudon KW, Riding AM, et al. Spatiotemporal immune zonation of the human kidney. Science. 2019 Sep;365(6460):1461-6.

45 Arazi A, Rao DA, Berthier CC, Davidson A, Liu Y, Hoover PJ, et al. The immune cell landscape in kidneys of patients with lupus nephritis. Nat Immunol. 2019 Jul;20(7):902-14.

46 Der E, Ranabothu S, Suryawanshi H, Akat KM, Clancy R, Morozov P, et al. Single cell RNA sequencing to dissect the molecular heterogeneity in lupus nephritis. JCI Insight. 2017 May;2(9):e93009.

47 Der E, Suryawanshi H, Morozov P, Kustagi M, Goilav B, Ranabothu S, et al. Tubular cell and keratinocyte single-cell transcriptomics applied to lupus nephritis reveal type I IFN and fibrosis relevant pathways. Nat Immunol. 2019 Jul;20(7):915-27.

48 Conway BR, O'Sullivan ED, Cairns C, O'Sullivan J, Simpson DJ, Salzano A, et al. Kidney single-cell atlas reveals myeloid heterogeneity in progression and regression of kidney disease. J Am Soc Nephrol. 2020 Dec; 31(12):2833-54.

49 Cameron GJM, Cautivo KM, Loering S, Jiang SH, Deshpande AV, Foster PS, et al. Group 2 innate lymphoid cells are redundant in experimental renal ischemia-reperfusion injury. Front Immunol. 2019;10:826.

50 Kreimann K, Jang MS, Rong S, Greite R, von Vietinghoff S, Schmitt R, et al. Ischemia reperfusion injury triggers CXCL13 release and Bcell recruitment after allogenic kidney transplantation. Front Immunol. 2020;11:1204.

51 Kirita Y, Wu H, Uchimura K, Wilson PC, Humphreys BD. Cell profiling of mouse acute kidney injury reveals conserved cellular responses to injury. Proc Natl Acad Sci U S A. 2020 Jul;117(27):15874-83.

52 Wen L, Yang H, Ma L, Fu P. The roles of NLRP3 inflammasome-mediated signaling pathways in hyperuricemic nephropathy. Mol Cell Biochem. 2021 Mar;476(3):1377-86

53 Nishinakamura R. Human kidney organoids: progress and remaining challenges. Nat Rev Nephrol. 2019 Oct;15(10):613-24.

54 Tran T, Lindström NO, Ransick A, De Sena Brandine $\mathrm{G}$, Guo $\mathrm{Q}$, Kim $\mathrm{AD}$, et al. In vivo developmental trajectories of human podocyte inform in vitro differentiation of pluripotent stem cell-derived podocytes. Dev Cell. 2019 Jul;50(1):102-e6.

55 Uchimura $\mathrm{K}, \mathrm{Wu} \mathrm{H}$, Yoshimura Y, Humphreys BD. Human pluripotent stem cell-derived kidney organoids with improved collecting duct maturation and injury modeling. Cell Rep. 2020 Dec;33(11):108514.

56 Subramanian A, Sidhom EH, Emani M, Vernon K, Sahakian N, Zhou Y, et al. Single cell census of human kidney organoids shows reproducibility and diminished off-target cells after transplantation. Nat Commun. 2019 Nov; 10(1):5462.

$57 \mathrm{Wu} \mathrm{H}$, Uchimura K, Donnelly EL, Kirita Y, Morris SA, Humphreys BD. Comparative analysis and refinement of human PSC-derived kidney organoid differentiation with single-cell transcriptomics. Cell Stem Cell. 2018 Dec;23(6):869-e8.

58 Czerniecki SM, Cruz NM, Harder JL, Menon R, Annis J, Otto EA, et al. High-throughput screening enhances kidney organoid differentiation from human pluripotent stem cells and enables automated multidimensional phenotyping. Cell Stem Cell. 2018 Jun;22(6): 929-e4.

59 Dvela-Levitt M, Kost-Alimova M, Emani M, Kohnert E, Thompson R, Sidhom EH, et al. Small molecule targets TMED9 and promotes lysosomal degradation to reverse proteinopathy. Cell. 2019 Jul;178(3):521-e23.

$60 \mathrm{Wu} \mathrm{H}$, Malone AF, Donnelly EL, Kirita Y, Uchimura K, Ramakrishnan SM, et al. Singlecell transcriptomics of a human kidney allograft biopsy specimen defines a diverse inflammatory response. J Am Soc Nephrol. 2018 Aug;29(8):2069-80.

61 Malone AF, Wu H, Fronick C, Fulton R, Gaut JP, Humphreys BD. Harnessing expressed single nucleotide variation and single cell RNA sequencing to define immune cell chimerism in the rejecting kidney transplant. J Am Soc Nephrol. 2020 Sep;31(9):1977-86.

62 Liu Y, Hu J, Liu D, Zhou S, Liao J, Liao G, et al. Single-cell analysis reveals immune landscape in kidneys of patients with chronic transplant rejection. Theranostics. 2020; 10(19):8851-62.

63 Wang Y, Zhao Y, Zhao Z, Li D, Nie H, Sun Y, et al. Single-cell RNA-Seq analysis identified kidney progenitor cells from human urine. Protein Cell. 2021 Apr;12(4):305-12.

64 Stark R, Grzelak M, Hadfield J. RNA sequencing: the teenage years. Nat Rev Genet. 2019 Nov;20(11):631-56.

65 Regev A, Teichmann SA, Lander ES, Amit I, Benoist C, Birney E, et al. The human cell atlas. Elife. 2017;12:6. 